Supporting information

\title{
Enhancing Photovoltaic Performance by Cathode Interfacial Modification with Inorganic/organic Gradient Diffusion Structures
}

Mingguang Li, 'Jing Li, ${ }^{\dagger}$ Jun Wang, Longsheng Yu, Tingting Wang, Jibiao Jin, Ligang Xu, Chao Zheng, Runfeng Chen* and Wei Huang

Key Laboratory for Organic Electronics and Information Displays \& Jiangsu Key Laboratory for Biosensors, Institute of Advanced Materials (IAM), Jiangsu National Synergetic Innovation Center for Advanced Materials (SICAM), Nanjing University of Posts \& Telecommunications, 9 Wenyuan Road, Nanjing 210023, China.

E-mail: iamrfchen@njupt.edu.cn

$\dagger$ These authors contributed equally to this work.

Total number of pages (including cover page): 8

Total number of Figures: 11

Total number of Tables: 4 

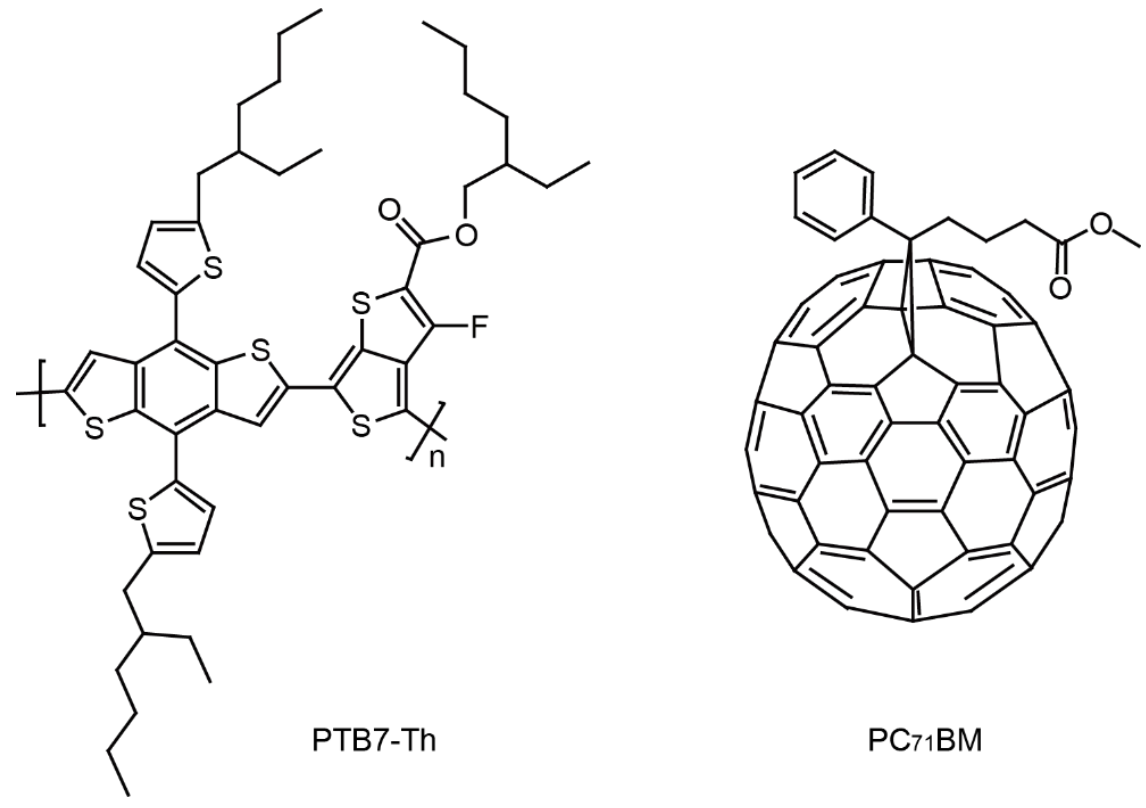

Scheme S1. Chemical structures of PTB7-Th and $\mathrm{PC}_{71} \mathrm{BM}$

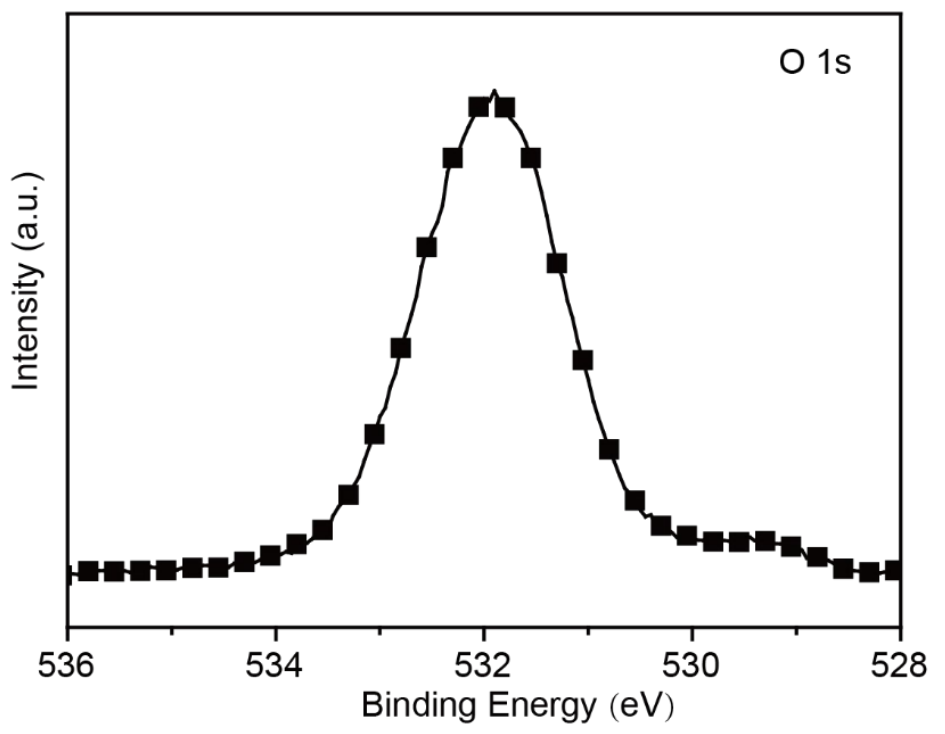

Figure S1. O 1s XPS spectrum of the pure ITIC film. 

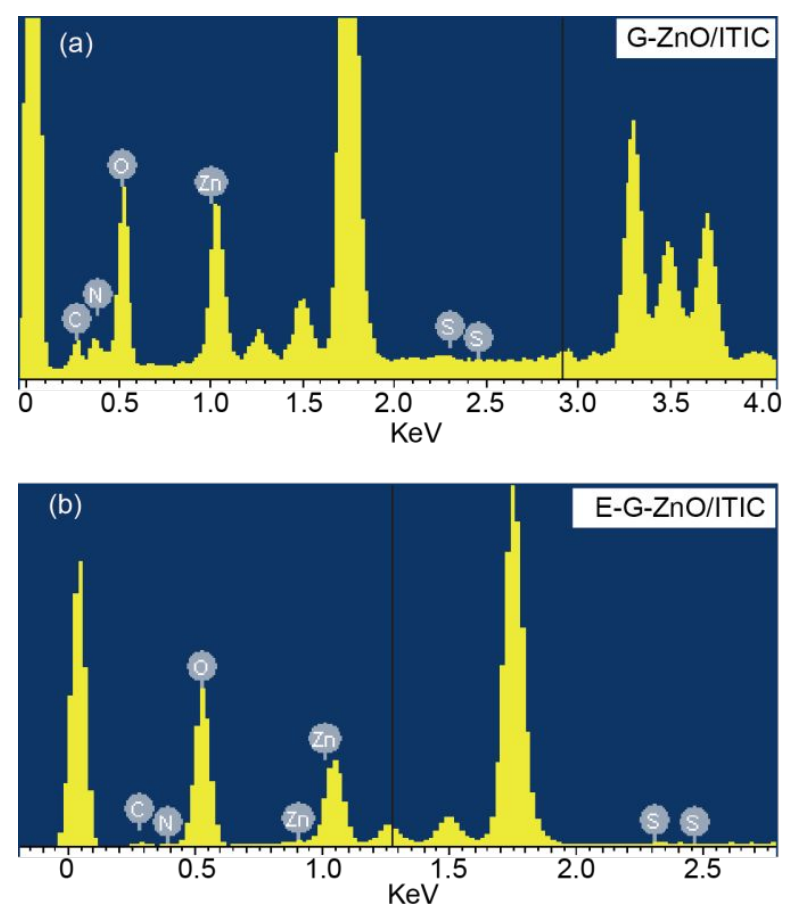

Figure S2. Elemental compositions of (a) G-ZnO/ITIC and (b) E-G-ZnO/ITIC layers.
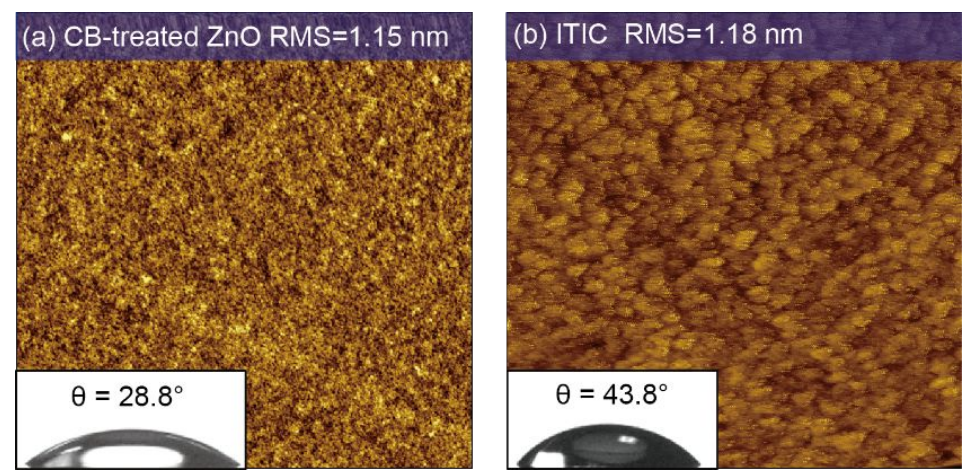

Figure S3. AFM height images $(5 \mu \mathrm{m} \times 5 \mu \mathrm{m})$ and corresponding contact angle images of (a) CB-treated $\mathrm{ZnO}$ and (b) pure ITIC layers spin-coated on ITO. 

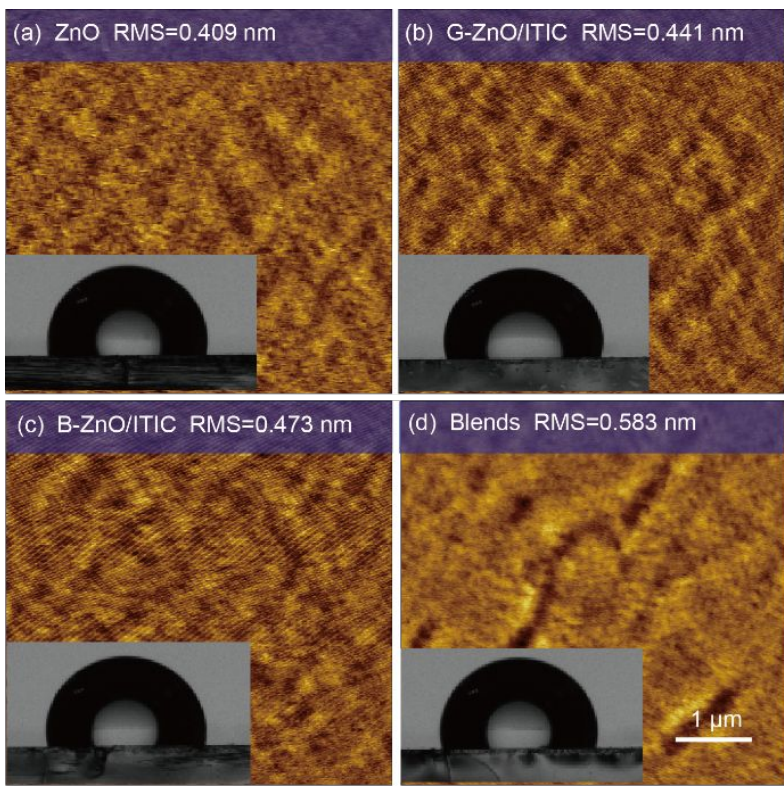

Figure S4. Film morphologies and contact angles of active layers fabricated on different CILs.

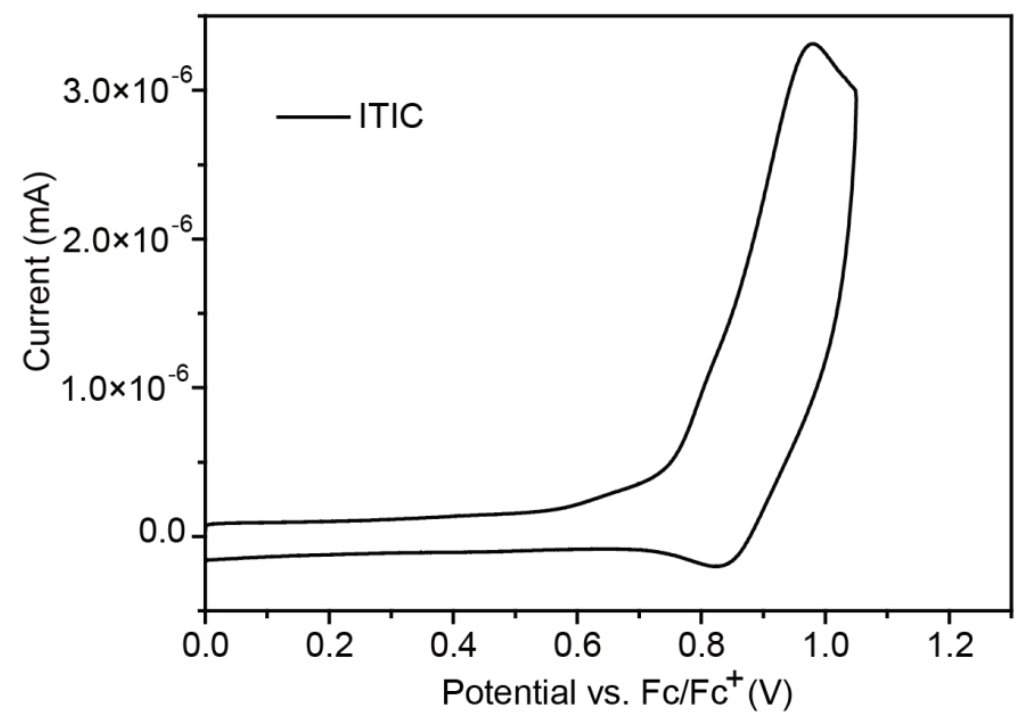

Figure S5. CV curve of the pure ITIC film. 


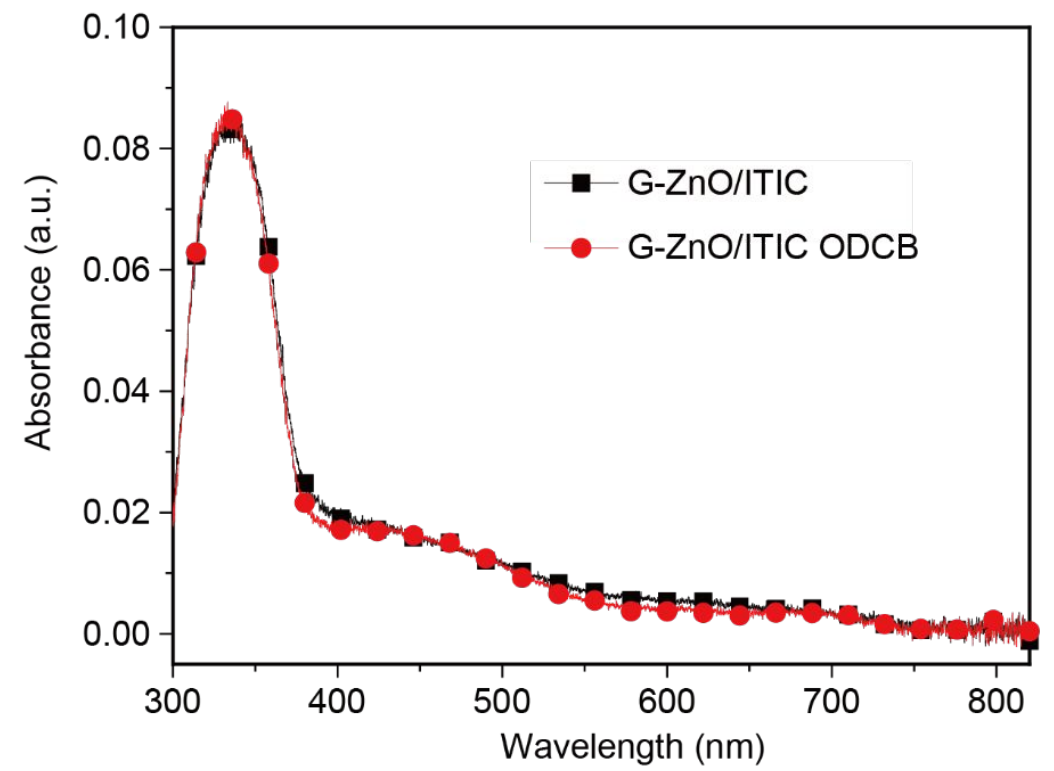

Figure S6. UV-visible absorption spectra of G-ZnO/ITIC CIL before and after spin-coating of ODCB solvent.

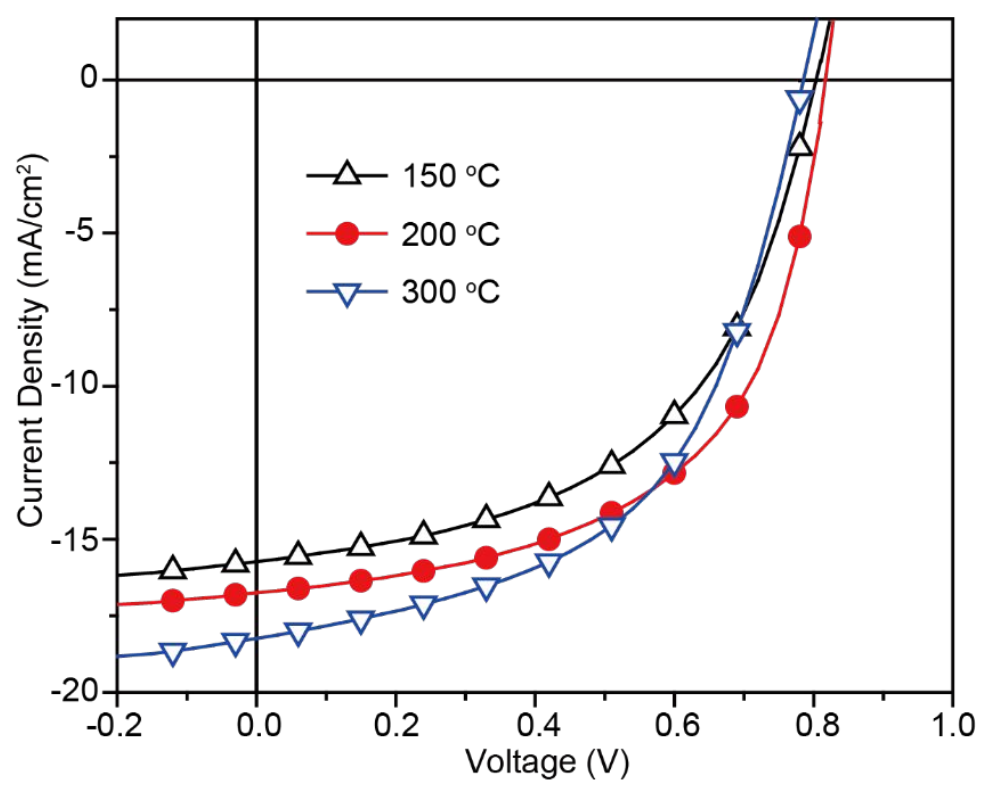

Figure S7. $J-V$ characteristics of the inverted PTB7-Th:PC ${ }_{71}$ BM-based OSCs with ZnO CILs, which were thermal annealed under different temperatures. 


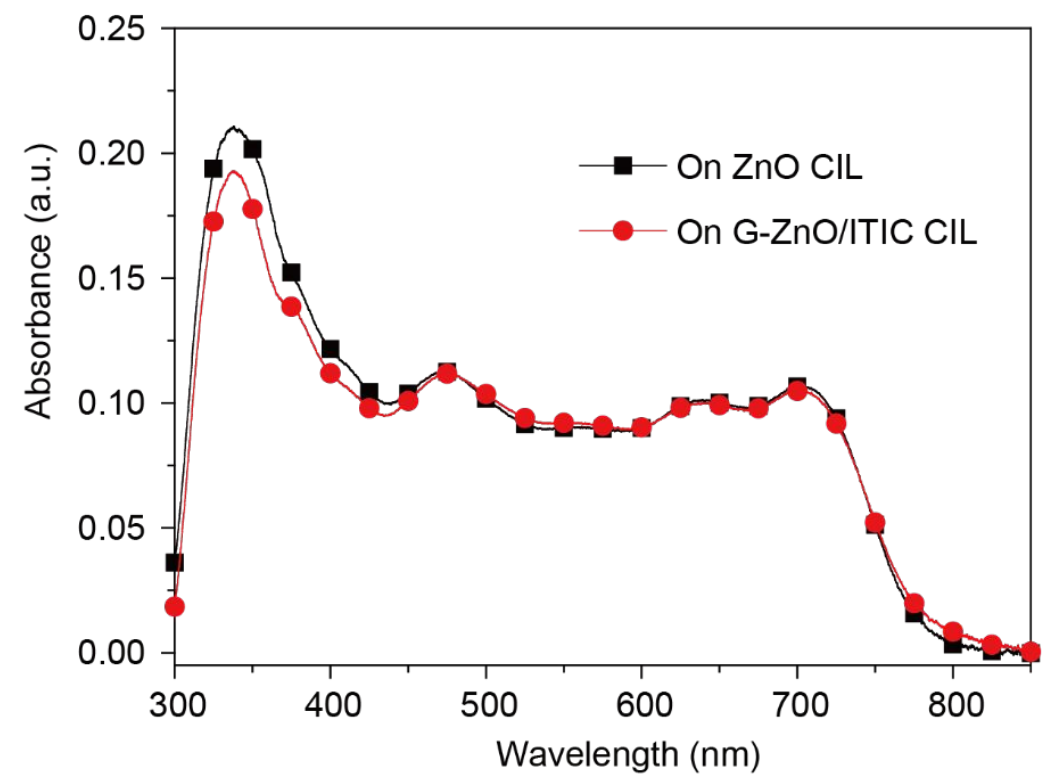

Figure S8. UV-visible absorption spectra of $\mathrm{PTB} 7-\mathrm{Th}: \mathrm{PC}_{71} \mathrm{BM}$ blend layers spin-coated on $\mathrm{ZnO}$ and G-ZnO/ITIC CILs, respectively.

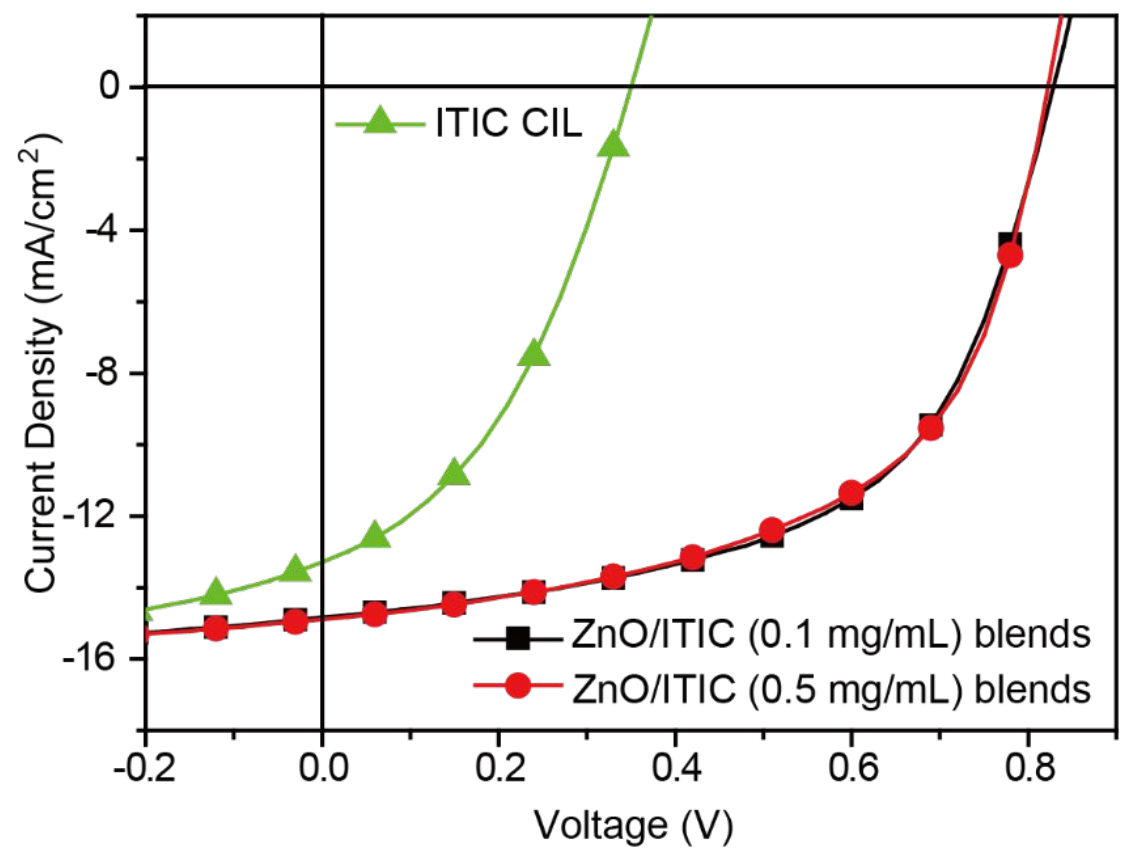

Figure S9. $J-V$ characteristics of the inverted $\mathrm{PTB} 7-\mathrm{Th}: \mathrm{PC}_{71} \mathrm{BM}$ solar cells with various CILs of pure ITIC layer, $\mathrm{ZnO} / \mathrm{ITIC}(0.1 \mathrm{mg} / \mathrm{mL})$ blend layer and $\mathrm{ZnO} / \mathrm{ITIC}(0.5 \mathrm{mg} / \mathrm{mL})$ blend layer under illumination of AM $1.5 \mathrm{G}$ at $100 \mathrm{~mW} / \mathrm{cm}^{2}$. 


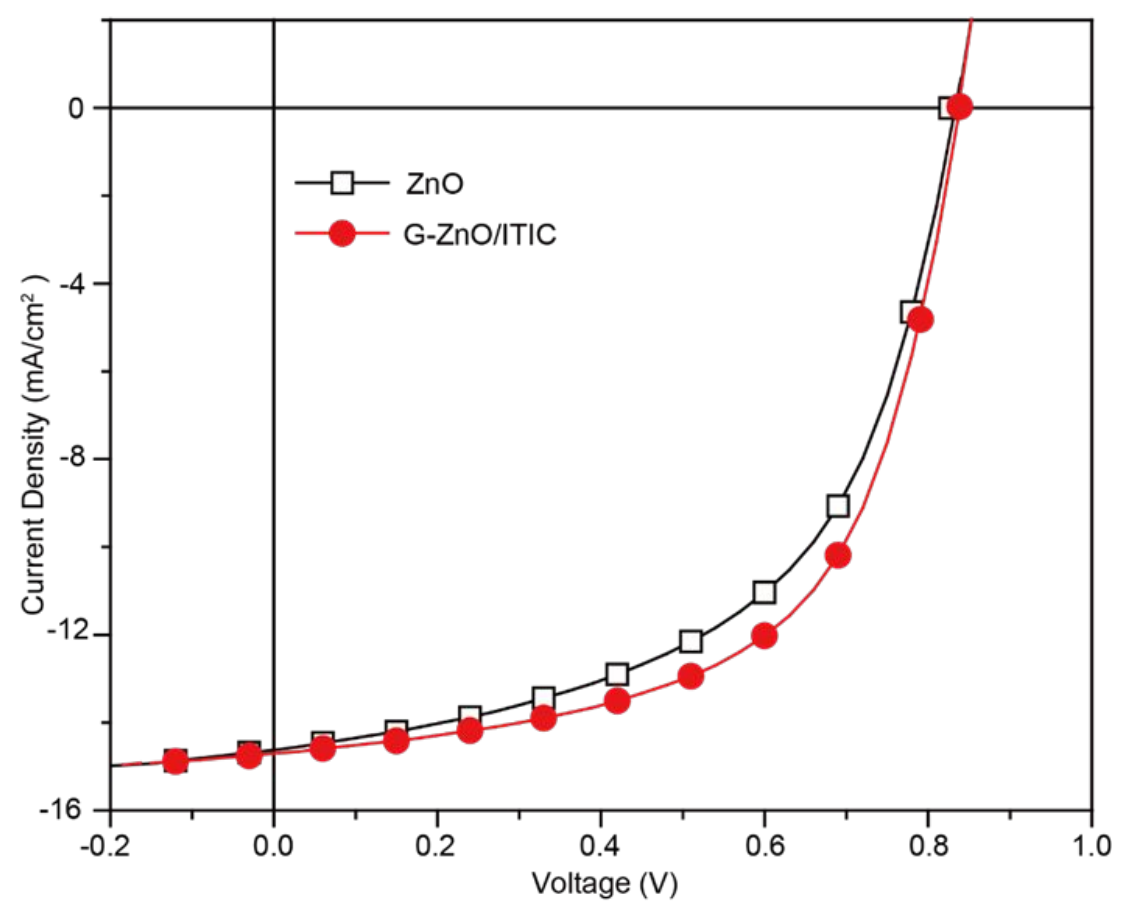

Figure S10. $J-V$ characteristics of the inverted PTB7-Th:ITIC-based non-fullerene OSCs with $\mathrm{ZnO}$ and $\mathrm{G}-\mathrm{ZnO} / \mathrm{ITIC}(1 \mathrm{mg} / \mathrm{mL})$ CILs.

Table S1 Detailed data of the fitting curves for O 1s XPS spectra.

\begin{tabular}{|c|c|c|c|c|c|c|}
\hline \multirow{2}{*}{$\begin{array}{l}\text { Types of } \\
\text { thin films }\end{array}$} & \multicolumn{3}{|c|}{ Peak 1} & \multicolumn{3}{|c|}{ Peak 2} \\
\hline & Position (eV) & $\operatorname{Area} \operatorname{ratio}\left(\mathrm{A}_{1}, \%\right)$ & FWHM & Position (eV) & Area ratio $\left(A_{1}, \%\right)$ & FWHM \\
\hline $\mathrm{ZnO}$ & 530.85 & 50.4 & 1.852 & 529.48 & 49.6 & 1.096 \\
\hline G-ZnO/ITIC & 530.87 & 66.2 & 2.147 & 529.73 & 33.8 & 1.044 \\
\hline E-G-ZnO/ITIC & 530.88 & 51.1 & 2.524 & 529.84 & 48.9 & 1.076 \\
\hline B-ZnO/ITIC & 531.19 & 57.0 & 1.957 & 529.92 & 43.0 & 1.067 \\
\hline ZnO/ITIC blends & 530.67 & 52.1 & 2.085 & 529.45 & 47.9 & 1.099 \\
\hline
\end{tabular}

Table S2. Elemental compositions (wt $\%$ ) of G-ZnO/ITIC and E-G-ZnO/ITIC layers.

\begin{tabular}{cccccccc}
\hline \multirow{2}{*}{ Type of CILs } & \multicolumn{9}{c}{ Wt\% } & \multirow{2}{*}{ N/Zn } \\
\cline { 2 - 6 } & $\mathbf{C}$ & $\mathbf{N}$ & $\mathbf{O}$ & $\mathbf{S}$ & $\mathbf{Z n}$ & \\
\hline G-ZnO/ITIC & 10.79 & 15.95 & 62.88 & 0.14 & 10.25 & 2.160 \\
E-G-ZnO/ITIC & 5.07 & 2.32 & 87.32 & 0.24 & 5.05 & 0.459 \\
\hline
\end{tabular}


Table S3 Performance of inverted PTB7-Th:PC ${ }_{71} \mathrm{BM}$ solar cells with CILs of pure ITIC layer and $\mathrm{ZnO} / \mathrm{ITIC}$ blend layer.

\begin{tabular}{ccccc}
\hline $\begin{array}{c}\text { Cathode interfacial layer } \\
(\mathbf{C I L})\end{array}$ & $\begin{array}{c}\boldsymbol{V}_{\mathbf{o c}} \\
(\mathbf{V})\end{array}$ & $\begin{array}{c}\boldsymbol{J}_{\mathbf{s c}} \\
\left(\mathbf{m A} \mathbf{/} \mathbf{c m}^{\mathbf{2}}\right)\end{array}$ & $\begin{array}{c}\mathbf{F F} \\
\mathbf{( \% )}\end{array}$ & $\begin{array}{c}\text { PCE } \\
\mathbf{( \% )}\end{array}$ \\
\hline $\mathrm{ITIC}$ & 0.33 & 13.28 & 42.6 & 1.87 \\
$\mathrm{ZnO} / \mathrm{ITIC}(0.1 \mathrm{mg} / \mathrm{mL})$ blends & 0.81 & 14.83 & 57.7 & 6.93 \\
$\mathrm{ZnO} / \mathrm{ITIC}(0.5 \mathrm{mg} / \mathrm{mL})$ blends & 0.81 & 14.89 & 56.8 & 6.85 \\
\hline
\end{tabular}

Table S4 Performance of inverted PTB7-Th:ITIC solar cells with CILs of pure ZnO layer and $\mathrm{G}-\mathrm{ZnO} / \mathrm{ITIC}$ layer.

\begin{tabular}{ccccc}
\hline $\begin{array}{c}\text { Cathode interfacial layer } \\
(\mathbf{C I L})\end{array}$ & $\begin{array}{c}\boldsymbol{V}_{\mathbf{o c}} \\
(\mathbf{V})\end{array}$ & $\begin{array}{c}\boldsymbol{J}_{\mathbf{s c}} \\
\left(\mathbf{m A} / \mathbf{c m}^{2}\right)\end{array}$ & $\begin{array}{c}\mathbf{F F} \\
\mathbf{( \% )}\end{array}$ & $\begin{array}{c}\text { PCE } \\
\mathbf{( \% )}\end{array}$ \\
\hline $\mathrm{ZnO}$ & 0.81 & 14.63 & 56.0 & 6.630 \\
$\mathrm{G}-\mathrm{ZnO} / \mathrm{ITIC}(1.0 \mathrm{mg} / \mathrm{mL})$ & 0.81 & 14.70 & 61.2 & 7.288 \\
\hline
\end{tabular}

